\title{
Geometric Correction in Recent High Resolution Satellite Imagery: A Case Study in Coimbatore, Tamil Nadu
}

\author{
Lt.Dr.S.Santhosh Baboo \\ Reader \\ Postgraduate and Research department of \\ Computer Science, \\ Dwaraka Doss Goverdhan Doss Vaishnav \\ College, Chennai
}

\author{
M.Renuka Devi \\ Asst.Prof \\ MCA Department, Sree Saraswathi \\ Thayagaraja College,Pollachi.
}

\begin{abstract}
Image Processing is a technique which is used to enhance raw images received from cameras and sensors placed on satellites, space probes and aircrafts or pictures taken in normal day-to-day life for various applications. Remotely-sensed data obtained from satellites or aircraft are usually geometrically distorted due to the acquisition system and the movements of the platform. A geometric correction of the image is required whenever the image is to be compared with existing maps or with other images. This paper deals with the composition of a correction function using ground control points. They offer a computational advantage and simplify the analysis of significant terms in the correction function. An accuracy analysis is performed, with emphasis being laid on the number and location of the ground control points.
\end{abstract}

Keywords:Geometric correction,Ground control points,Image Processing.

\section{INTRODUCTION}

Preprocessing of satellite images prior to image classification and change detection is essential. Preprocessing commonly comprises a series of sequential operations, including atmospheric correction or normalization, image registration, geometric correction, and masking (e.g., for clouds, water, irrelevant features)

The normalization of satellite imagery takes into account the combined, measurable reflectances of the atmosphere, aerosol scattering and absorption, and the earth's surface. It is the volatility of the atmosphere which can introduce variation between the reflectance values or digital numbers (DN's) of satellite images acquired at different times. Although the effects of the atmosphere upon remotely sensed data are not considered errors, since they are part of the signal received by the sensing device, consideration of these effects is important. The goal conveniently should be that following image preprocessing, all images should appear as if they were acquired from the same sensor.

The goal of image rectification is to facilitate the overlay of additional imagery and other geographic data sets. A standard map area, with boundaries set in UTM, is established for each scene, thus all image files for the same region, once rectified, will occupy the same map area. The UTM bounds for the scene are established according to the file size, the $28.5 \times 28.5 \mathrm{~m}$ pixels, and the minimum/maximum northing and easting required to contain the full scene area. These boundaries, the UTM zone and the ellipsoid are established on each newly- created empty file.
Geometric rectification of the imagery resamples or changes the pixel grid to fit that of a map projection or another reference image. This becomes especially important when scene to scene comparisons of individual pixels in applications such as change detection are being sought.

In some cases, Landsat TM scenes are much larger than a project study area. In these instances it is beneficial to reduce the size of the image file to include only the area of interest. This not only eliminates the extraneous data in the file, but it speeds up processing due to the smaller amount of data to process. This is important when utilizing multiband data such as Landsat TM imagery. This reduction of data is known as subsetting. This process cuts out the preferred study area from the image scene into a smaller more manageable file.

Once the raw remote sensing digital data has been acquired, it is then processed into usable information. Analog film photographs are chemically processed in a darkroom whereas digital images are processed within a computer. Processing digital data involves changing the data to correct for certain types of distortions. Whenever data is changed to correct for one type of distortion, the possibility of the creating another type of distortion exists. The changes made to remote sensing data involve two major operations: preprocessing and postprocessing.

The following Coimbatore imagery in Figure 1 shows the necessary of preprocessing. Because the Coordinates not in British National Grid coordinates.

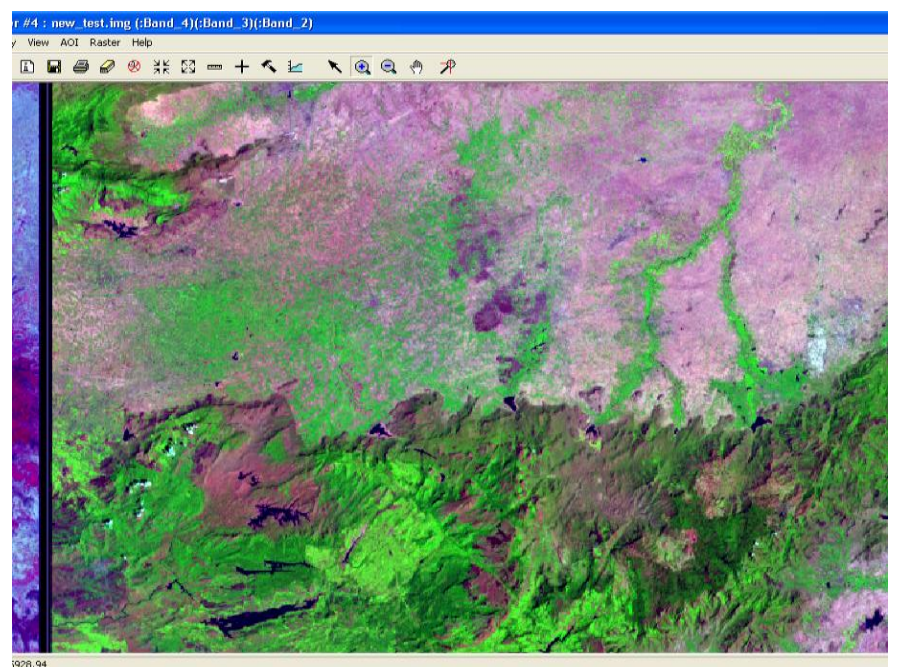

Figure 1.Geometrically not corrected Coimbatore Imagery 
The preprocessing steps of a remotely sensed image generally are performed before the postprocessing enhancement, extraction and analysis of information from the image. Typically, it will be the data provider who will preprocess the image data before delivery of the data to the customer or user. Preprocessing of image data often will include radiometric correction and geometric correction. But this paper only focus on geometric correction.

- Radiometric corrections are made to the raw digital image data to correct for brightness values, of the object on the ground, that have been distorted because of sensor calibration or sensor malfunction problems. The distortion of images is caused by the scattering of reflected electromagnetic light energy due to a constantly changing atmosphere. This is one source of sensor calibration error.

- Geometric corrections are made to correct the inaccuracy between the location coordinates of the picture elements in the image data, and the actual location coordinates on the ground. Several types of geometric corrections include system, precision, and terrain corrections.

\section{GEOMETRIC CORRECTION}

Geometric correction is necessary to preprocess remotely sensed data and remove geometric distortion so that individual picture elements (pixels) are in their proper planimetric $(x, y)$ map locations. This allows remote sensing-derived information to be related to other thematic information in GIS. Geometrically corrected imagery can be used to extract accurate distance, polygon area, and direction (bearing) information.

Remotely sensed imagery collected from airborne or spaceborne sensors often contain internal and external geometric errors. These can be systematic (predictable)or nonsystematic (random).Systematic geometric error is generally easier toidentify and correct than random geometric error. Some of these errors can be corrected by using ephemeris of the platform and known internal sensor distortion characteristics. Other errors can only be corrected by matching image coordinates of physical features recorded by the image to the geographic coordinates of the same features collected from a map or global positioning system (GPS).

Internal geometric errors are introduced by the remote sensing system itself or in combination with Earth rotation or curvature characteristics. These distortions are often systematic (predictable) and may be identified and corrected using pre-launch or in-flight platform ephemeris (i.e., information about the geometric characteristics of sensor and the Earth at data acquisition).

Geometric distortions in imagery that can sometimes be corrected through analysis of sensor characteristics and ephemeris data include:

- skew caused by Earth rotation effects,

- scanning system-induced variation in ground resolution cell size, relief displacement, and tangential scale distortion.

External geometric errors are usually introduced by phenomena that vary in nature through space and time. The most important external variables that can cause geometric error in remote sensor data are random movements by the aircraft (or spacecraft) at the exact time of data collection, which usually involve:
- altitude changes, and/or

- attitude changes (roll, pitch, and yaw).

In order for a GIS software package to correctly display a raster or vector image the image must contain geospatial information. The geospatial information relates the image pixel coordinate values to real world coordinate values (e.g., the British National Grid). The geospatial information enables the software to display the images with the correct scaling and orientation when viewed in conjunction with other GIS data, such as a DEM.

Aerial photographs are not easily Geo Correcting. Image orthorectification must be conducted to remove Spherical lens distortion and topographic relief offset of ground based features. However, Geo Correcting of a map extract is a straightforward procedure as the image is essentially planimetrically correct, that is the length scale remains constant over the entire image area.To do geo Correcting your scanned field slip you will input four control points with known $\mathrm{x}$ and $\mathrm{y}$ coordinates into the corners of the images. The coordinate values used will be junctions of N-S and E-W oriented divisions of the British National grid, which can be assumed to have a precise position and value.

There are different levels of geometric correction of remotely sensed imagery: The first level is Registration.In this stage the alignment of one image to another image of the same area.The next step is Rectification. This means alignment of image to a map so that the image is planimetric, just like the map. Also known as geo-referencing. The next level is Geocoding. A special case of rectification that includes scaling to a uniform standard pixel GIS. The use of standard pixel sizes and coordinates permits convenient layering of images from different sensors and maps into a GIS. The final level is Orthorectification. This level focus correction of the image, pixel by pixel for topographic distortion. The result is that every pixel appears to be viewing the earth from directly above, i.e. the image is in a strict orthographic projection.

Generaly there are two strategies for geometric correction: First one is modelling the nature and magnitude of all sources of geometric distortion and find the corresponding correction formulae. This approach works well when all sources of distortion are well understood and can be quantified. This is not the case for all sources of distortion, particularly not for the unsystematic variations in satellite altitude etc. The second stragiey is "Empirical" approach, using reference points (ground control points=GCPs).

The model required for geometric correction is then built from the positional differences between reference points and their location in the imagery. This approach does not require knowing either the source or the magnitude of the errors. It is, therefore, the more frequently applied approach.

Basically there are two types of geocorrection to be carried out.First one is Image to Map geocorrection and another is image to image geocorrection. This paper only focus on the first mehtod. For the Image to Map geocorrection system there are two basic operations that must be performed to geometrically rectify a remotely sensed image -

i). Development of Mathematical Transformation between image and map and ii).Pixel Interpolation. 


\section{IMAGE TO MAP GEOCORRECTION}

Geometric distortions introduced by distortions can be corrected using ground control points and appropriate mathematical models. A ground control point (GCP) is a location on the surface of the Earth (e.g., a road intersection) that can be identified on the imagery and located accurately on a map.It is show in the Figure 3 below.

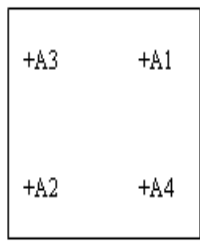

MMAG

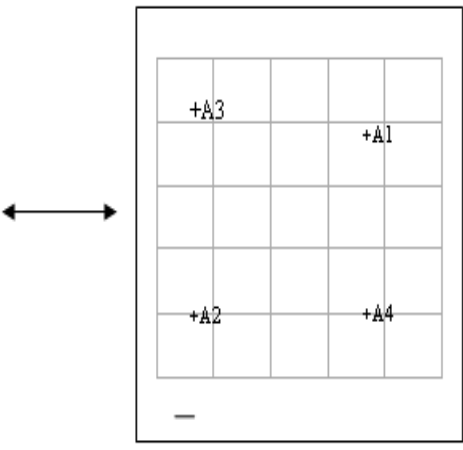

MAP
Figure 3.Image to Map Geocorrection

The image analyst must be able to obtain two distinct sets of coordinates associated with each GCP:

- image coordinates specified in i rows and $\mathrm{j}$ columns, and

- map coordinates (e.g., $x, y$ measured in degrees of latitude and longitude, feet in a state plane coordinate system, or meters in a Universal Transverse Mercator projection).

The paired coordinates from many GCPs (e.g., 20) can be modeled to derive geometric transformation coefficients. These coefficients may be used to geometrically rectify the remote sensor data to a standard datum and map projection. The GCPs should be spread evenly over the image, covering the whole image, and be placed as much as possible into the corners of the image, to give best coverage for calculating the transformation.

This is not he case in the provided data, as no corners are used, and there is a slight over-representation of GCP in the lower half of the image. Unfortunately, adding GCP from the given map is also limited, as the map covers much less area than the actual image. Clustering GCPs in the centre of the image will not give accuracy to the transformation of coordinates in the extremities of the image.

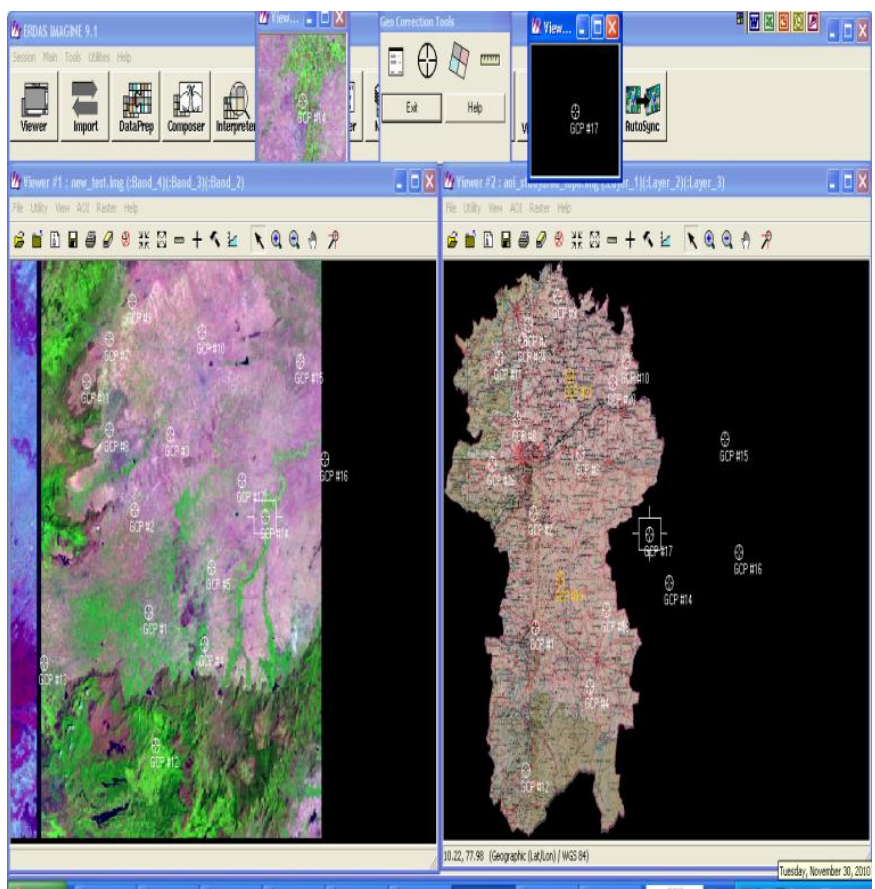

Figure 3.1 Geocorrection using Coimbatore imagery and Coimbatore Toposheets.

The above Figure 3.1 explain the image to map geocorrection and we can see the ground control point spread all over the map.Right side of the image is study area of the Coimbatore toposheets.Adding GCP from a map is easy and straightforward. However, care should be taken when choosing the correct position of a GCP, as a scanned map might add to the RMS error, because roads tend to be drawn wider than they actually are, or even slightly displaced, i.e. University Road is said to be approximately $30 \mathrm{~m}$ wide. In addition, the pixel size of the map is $3 \mathrm{~m}$; the image itself operates with $30 \mathrm{~m}$, which is another source of error in correct placement of GCP.

\section{IMAGE-TO-IMAGE GEOCORRECTION}

Image-to-image registration is the matching of one image to another so the same geographic area is positioned coincident with respect to the other. This type of geometric correction is used when it is not necessary to have each pixel assigned a unique $x, y$ coordinate in a map projection. For example, we might want to make a cursory examination of two images obtained on different dates to see if any change has taken place. The following Figure 4 shows the image to image geocorrection,here image 1 is to be geocorrected and image 2 is same area of image in previous year. 


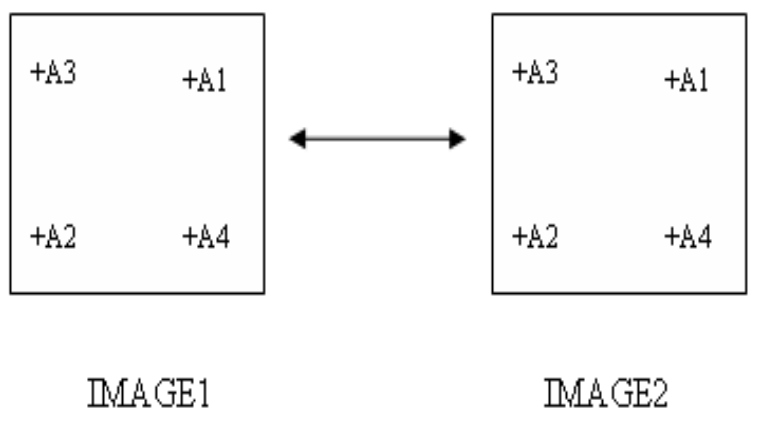

Figure 4.Image to Image Geocorrection

\section{DEVELOPMENT OF MATHEMTICAL TRANSFORMATION}

Equations need to be fitted to the GCP data using leastsquares criteria to model the corrections. Depending on the distortion in the imagery, the number of GCPs used, and the degree of topographic relief displacement in the area, higher-order polynomial equations may be required to geometrically correct the data.The order of the rectification is simply the highest exponent used in the polynomial. Calculate new output pixel locations (X, $\mathrm{Y})$ and relate image location to map location using a "mapping polynomial" function

$$
\begin{aligned}
& \mathrm{X}^{\prime}=\mathrm{a} 0+\mathrm{a} 1 \mathrm{X}+\mathrm{a} 2 \mathrm{Y}+\mathrm{a} 3 \mathrm{XY}+\mathrm{a} 4 \mathrm{X} 2+\mathrm{a} 5 \mathrm{Y} 2 \\
& Y^{\prime}=b 0+b 1 X+b 2 Y+b 3 X Y+b 4 X 2+b 5 Y 2
\end{aligned}
$$

Using these mapping functions calculate correct map locations (X', Y') for input pixel locations (X, Y) Before applying the coefficients to create the rectified output image, it is important to determine how well the transformation derived from the leastsquares regression of the GCPs account for the geometric distortion in the input image. The method used most often involves the computation of the root-mean-square error (RMSerror) for each of the ground control points.

Selecting GCPs and deriving the transformation and RMS error is an iterative process.

- First, an initial set of GCPs (e.g., 20) are used to compute an initial set of coefficients and constants.

- The root mean squared error (RMSE) associated with each of these initial 20 GCPs is computed and summed.

- Then, the individual GCPs that contributed the greatest amount of error are determined and deleted.

- After the first iteration, this might only leave 15 of 20 GCPs.

A new set of coefficients is then computed using the 16 GCPs. The process continues until the RMSE reaches a user-specified threshold (e.g., $<1$ pixel error in the $\mathrm{x}$-direction and $<1$ pixel error in the y-direction). The main goal of GCP is to remove the GCPs that introduce the most error into the multiple-regression coefficient computation. When the acceptable threshold is reached, the final coefficients and constants are used to rectify the input image to an output image in a standard map projection.
Table 5.Ground Control Points.Control Point Error:X:0.4965

\begin{tabular}{|c|c|c|c|c|c|c|c|c|c|c|c|c|c|}
\hline$\angle C O P T O C$ & llopot: ine & timg| Re & cencer: testin & (eff,gec) & & & & & & & & & 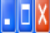 \\
\hline Fie Ven: & & & & & & & & & & & & & \\
\hline 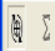 & घ & i & 112 & $\cos 1 \mathrm{Pu}^{2}$ & vilers: $M O$ & 85 MDOSK5 & :10685 & & & & & & \\
\hline Pont: & Povillo & $3 \mathrm{Cba}$ & Xlad & Ylood & > Cod & XPBd & YPÁ. & Tper & XPeridas & YPeidis & PMSEIII & Conth Hito & ath 1 \\
\hline 1 & 6PPH & & 265540 & 30150 & & 77006 & 10653 & Cortol & 2480 & 228 & 0505 & 098 & \\
\hline 2 & 60912 & & 256083 & .2519806 & & 7700 & 10808 & Cartal & 1201 & 2.330 & 24835 & 0814 & \\
\hline 3 & GPB & & 34.801 & 1781.30 & & 77.182 & 11013 & \begin{tabular}{|l|l|l|l|} 
\\
\end{tabular} & 2400 & 0273 & 0,552 & 0912 & \\
\hline 4 & GPF & & 327.74 & .3147765 & & 77.28 & 10068 & Cortal & ABAP & 2.30 & 093 & 154 & \\
\hline 5 & GP\# & & 32200 & 3932158 & & 77227 & 10531 & Cortal & 0181 & 1000 & 019:5: & 0.32 & \\
\hline 6 & GPP & & 1875674 & 17786.65 & & 76940 & $11: 08$ & Cortal & 0225 & 0.30 & 045 & 075 & \\
\hline 1 & 60P77 & & 187300 & Wu1313 & & 76980 & 1128 & Cortol & 0240 & 0.34 & Q445: & 0720 & \\
\hline 8 & QDP $\$ 10$ & & 34000 & .92272 & & 77.35 & 11201 & Cortold & . 2351 & 1200 & Q477 & 0.87 & \\
\hline 9 & GPP 10 & & 2919068 & 477000 & & 770.05 & 11330 & Cortod & 0200 & $d 1 x$ & 022 & (040) & \\
\hline 10 & QDSHII & & 148000 & 120028 & & 76872 & 1120 & Cortold & 0179 & 248 & 0527] & 0871 & \\
\hline 11 & OPNA12 & & 2761.163 & 432000 & & 76973 & 1059 & Cortal & D.887 & 2002 & 086 & 1.40 & \\
\hline 12 & QDP $\# 13$ & & 6632 & 4127,750 & & 7650 & 1064 & Cortol & 01160 & a017 & Q167 & 0286 & \\
\hline 13 & QDPA14 & & 452115 & 2069808 & & 77.52 & 1074 & Cortal & A813 & 0556 & 0.55 & 158 & \\
\hline 14 & OQP $\# 15$ & & 52280 & 1165.24 .2 & & 77.76 & 11008 & \begin{tabular}{|c|c|c|} 
Cartol \\
\end{tabular} & 070 & 0336 & 078 & 1200 & \\
\hline 15 & QDP $\$ 16$ & & 5908083 & 2066000 & & 77.79 & 10008 & Cortol & 0670 & 2060 & 090 & 1460 & \\
\hline 16\lceil & QDP $\$ 17$ & & 441420 & 2297.06 & & 77.47 & 1084 & Cortol & (2198 & 0224 & 036 & $\mid 057$ & v \\
\hline$k$ & & & & & & & & & & & & & ? \\
\hline
\end{tabular}
Y:0.3465 RMS Error Total:0.60

The above Table 5 shows the 16 ground control points in the Coimbaore imagery to map georeference and it proves the minimum RMS error .we have maintain <1 RMS error. In this geocorrection RMS error is 0.6 so it is below the one.So it will provide the high quality gereferenced image.

\section{PIXEL INTERPOLATION}

First the pixel brightness values must be determined. As there may be no direct one-to-one relationship between the movement of input pixel values to output pixel locations, the rectified output image often requires a value from the input pixel grid that does not fall neatly on a row-and-column coordinate. When this occurs, there must be some mechanism for determining the brightness value to be assigned to the output rectified pixel. This process is called pixel interpolation.

In order to geometrically correct the original distorted image, a process called resampling is used to determine the digital values to place in the new pixel locations of the corrected output image.

The resampling process calculates the new pixel values from the original digital pixel values in the uncorrected image. There are three common methods for resampling:

- nearest neighbour, bilinear interpolation, $\bullet^{\bullet}$ and cubic convolution. The following Figure 6 shows the resampling imagery of coimbatore using nearest neighbour resampling mehtod.

\section{RESULT AND DISCUSSION}

There are two methods are used to check the image is correctly georeferenced. When we reopen the image in the viewer in ERDAS Imagine.when you move the cursor around the image the position values given at the bottom left of the window should be British National Grid coordinates,Projection type, WGS84 is Spheroid and Datum and not image pixel coordinates.It is show in the following Figure 7. 


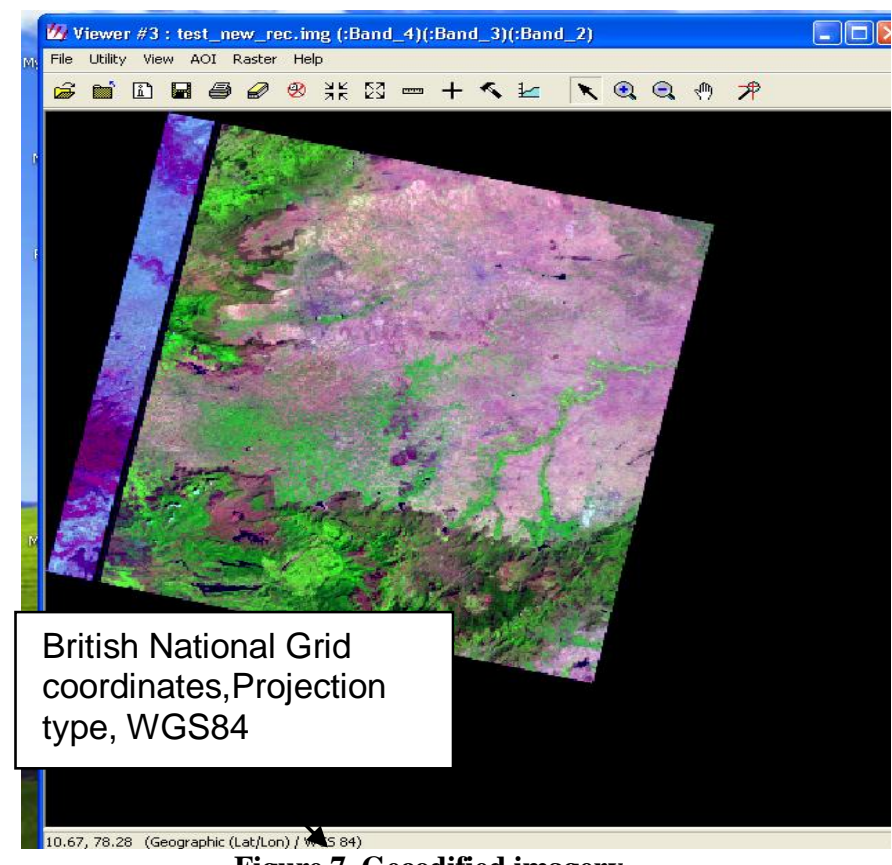

Figure 7. Gecodified imagery.

In some cases it is necessary to mosaic a number of individual scenes. To do this the scenes should be rectified to the same map projection and datum. One of the images to be mosaicked is designated as the base image. The base image and rectified image will normally overlap a certain amount (e.g., $20 \%$ to 30\%).we can see the edge matching and river mathing by overlapping these two image. We can see these matching in the following figure.7.1.In this coimabtore settlement is match with each other. Here the base image is coimbatore map another is rectified image.

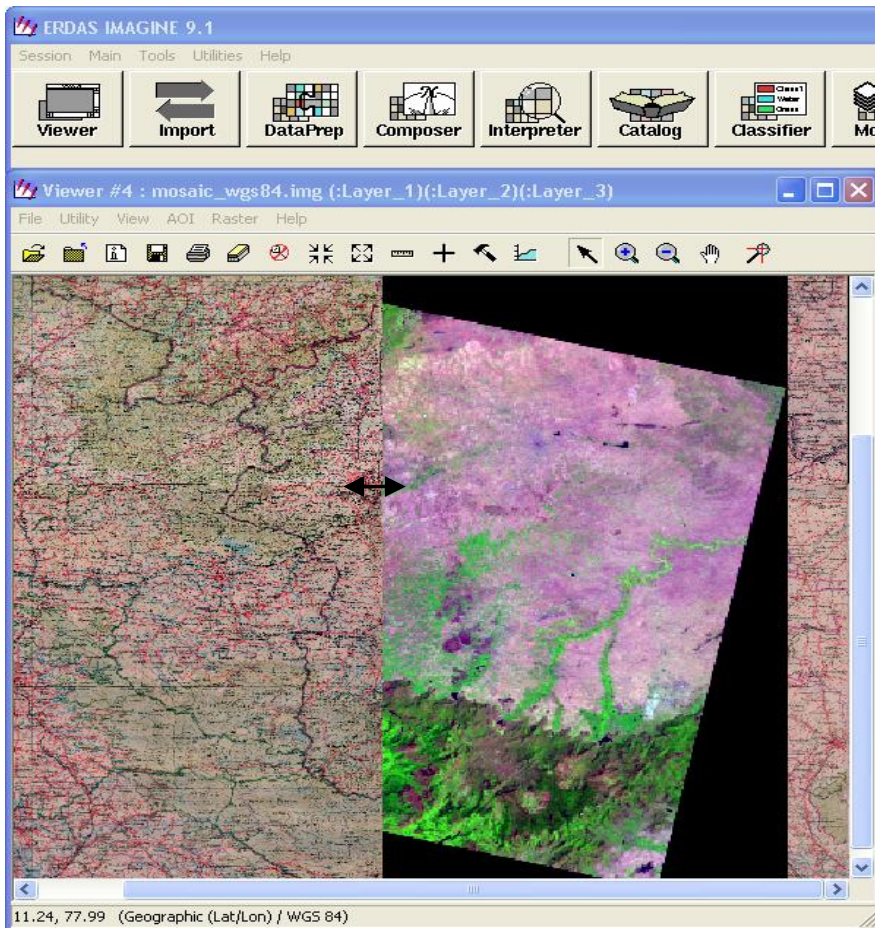

Figure 7.1 Image Mosaic process

\section{CONCLUSION}

The preprocessing of remotely sensed image is very imaportant to improve the quality and to remove the errors. It consists of two correction geometric and radiometric correction. This paper only focus on geometric correction. This geometric correction use Geographic (Lat/Lon) as projection type WGS84 is Spheroid and Datum and polynomial geometric method. This paper shows the geometric correction process done in the Coimbatore imagery to improve the quality and it shows the process of distortion removed .Finally we get the georectified image using ERDAS Imagine 9.1. It shows in figure 7.

\section{REFERENCES}

[1] Dowman,I., and Tao C.V.,2002.An update on rational functions for Photogrammetric restitution,Highlights ISPRS,vol.7,No.3,pp.22-29.

[2] Fraser,C.S.,Baltsavias, E.,Gruen, A.,2002.Processing of Ikonos imagery for submetre $3 \mathrm{D}$ positioning and building extraction,ISPRS Journal of photogrammetry \& Remote Sensing 56 (2002) 177-194.

[3] Fraser ,C., Hanley, H., Yamakawa, T.2001.sub-Metre Geopositioning with Ikonos Geo imagery, ISPRS Joint Workshop on High Resolution Mapping from Space, Hanover,Germany ,2001.

[4] Fritz,L.,1995.Recent Developments for Optical Earth Observation in the United states, Photogrammetric Week,pp75-84,Stuttgart ,1995.

[5] Hanley,H.B. and Fraser,C.S.,2001.Geopositioning accuracy of Ikonos imagery : indications from 2D transformations,photogrammetric Record ,17(98) : 317329.

[6] Hattori,S.,one ,T., Fraser, C.S. and Hasegawa,H.,2000. Orientation of high-resolution satellite images based on affine Projection,International Archires of photogrammetry \&Remote Sensing, Amsterdam,33(B3):59- 366

[7] Li, R., Zhou, G., Yang, S., Tuell, G., Schmidt, N. J. and Flower, C , 2000, Astudy of the potential attainable geometric accuracy of IKONOS satellite images, IAPRS, 33(B4), 587-595.

[8] Mikhail, E.M., Bethel, J.S. and McGlone, J.C., 20001, Itroduction to modern photogrammetry, John Wiley\&sons, New York.

[9] Digital Image Processing - A Remote Sensing Perspective,Jhon R. Jenson, $3^{\text {rd }}$ Edition, Prentice - Hall, 2003.
Digital Image Processing - Kenneth R. Castleman,

KMM Rao, Medical Image Processing, Proc. of workshop on Medical Image Processing and Applications, 8 th October 1995 @ NRSA, Hyderabad37.

KMM Rao, Image Processing for Medical Applications, Proc. of $14^{\text {th }}$ world conference on NDT, $8^{\text {th }}-13^{\text {th }}$ Dec 1996.

Ramanjaneyulu M, KMM Rao, A Novel technique to Resample High Resolution Remote Sensing Satellite Images, Proc. of IGRASS-02, Colorado. KMM et al., Design and Fabrication of Color Scanner, Prentice- Hall, 1996 
Indian Journal of Technology, Vol 15, Apr 1997. Fundamentals Of Digital Image Processing - Anil K. Jain, Prentice-Hall, 1989.

\section{Bibiliography:}

Lt.Dr.S.Santhosh Baboo, has around Seventeen years of postgraduate teaching experience in Computer Science, which includes Six years of administrative experience. He is a member, board of studies, in several autonomous colleges, and designs the curriculum of undergraduate and postgraduate programmes. He is a consultant for starting new courses, setting up computer labs, and recruiting lecturers for many colleges. Equipped with a Masters degree in Computer Science and a Doctorate in Computer Science, he is a visiting faculty to IT companies. It is customary to see him at several national/international conferences and training programmes, both as a participant and as a resource person. He has been keenly involved in organizing training programmes for students and faculty members. His good rapport with the IT companies has been instrumental in on/off campus interviews, and has helped the post graduate students to get real time projects. He has also guided many such live projects.
Lt.Dr. Santhosh Baboo has authored a commendable number of research papers in international/national Conference/journals and also guides research scholars in Computer Science. Currently he is Reader in the Postgraduate and Research department of Computer Science at Dwaraka Doss Goverdhan Doss Vaishnav College (accredited at 'A' grade by NAAC), one of the premier institutions in Chennai.

Mrs. M.Renuka Devi, has nearly 8 years of post graduate teaching experience in Computer Science. She has indulged in training the post graduate students to complete real time projects and also guides research scholars in Computer Science. Currently she is working as Lecturer in the Department of MCA at Sree Saraswathi Thyagaraja College (Autonomous),and An ISO 9001 Certified / NAAC Accredited Institution, Pollachi, Coimbatore (Dt), Tamil Nadu,India. 